\title{
Vacío institucional y construcción mediática de la sexualidad adolescente en Canadá y Chile: el imaginario del riesgo ${ }^{1}$
}

\section{Andrea Martínez}

Universidad de Ottawa, Canada. School of International Development \& Global Studies

\author{
Aldo Meneses
}

Universidad de Chile. Instituto de Asuntos Públicos

Recibido: 13-02-2007

Aceptado: $15-10-2008$

\section{Resumen}

Este artículo compara las narrativas de las diversas expresiones de la sexualidad adolescente a partir de una muestra de diarios de amplia circulación en Canadá y Chile, dos sociedades abiertas a procesos de cooperación e intercambio comercial e intercultural pese a estructuras jurídico-políticas, tradiciones y niveles de desarrollo diferentes. El análisis de ciertos ejes discursivos dominantes como son: riesgos asociados a la práctica de la sexualidad, legitimación de la anticoncepción como reacción al abuso sexual y autoagresiones entre los adolescentes, revela una perspectiva catastrofista de la sexualidad adolescente, conformando así un vacío informativo que limita el debate en torno a quien (estado, educadoras, padres, estudiantes) debe asumir un rol determinante en la producción y divulgación de estos contenidos. Frente al vacío institucional, emerge una respuesta adolescente que evidenciando las dudas propias de este estadio del desarrollo personal demuestra apertura a la existencia y manifestación de variadas identidades sexuales que sobrepasan la versión estrictamente dicotómica de lo masculino y femenino. Esto por sí mismo subvierte el imaginario del riesgo que recoge la prensa de mayor difusión en ambos países.

Palabras clave: medios de comunicación, sociología de la juventud, prácticas sexuales, imaginarios sociales.

1. Este trabajo forma parte de una investigación denominada Comportamientos sexuales de los adolescentes: aspectos educativos y sociopolíticos en Chile y Canadá, que, desde el año 2004, llevan a cabo sus autores a través de un convenio suscrito entre el Instituto de la Mujer de la Universidad de Ottawa, Canadá, y el Inap de la Universidad de Chile. Esta investigación cuenta con varias otras publicaciones en Chile y en el extranjero. En este caso, se analizaron las publicaciones de dos medios de amplia circulación en cada país durante un mes. Esta revisión se inició confeccionando una lista de términos descriptores relativos a la sexualidad adolescente que sirvieron para seleccionar las noticias que los periódicos consignaban al respecto. Éstas fueron posteriormente escaneadas y tratadas mediante un análisis de tipo factorial informatizado, el cual mostró los diversos cruces de sentido y las conjunciones del mismo, a partir de lo cual se elaboró el presente artículo. 
Abstract. Institutional Vacuum and Media Construction of Adolescent Sexuality in Canada and Chile: The Risk Imaginary

This paper compares the media coverage of adolescent sexuality's narratives conveyed by a sample of mainstream newspapers in Canada and Chile, two societies committed to enhance their commercial and intercultural cooperation in spite of different juridical and political structures, traditions and developmental levels. The analysis of discursive dominant axes such as the risks associated with sexual practice, the legitimization of contraception in reaction to sexual abuse, or self-aggressions among teens reveals an informative vacuum triggered by a catastrophist perspective of adolescent sexuality that limits the debate about who (the state, educators, parents, students) must assume a determinant role in the production and outreach of such contents. In response to this institutional vacuum, an alternative teen path emerges that not only highlights the doubts inherent at this stage of the personal development, but also welcomes the existence and manifestation of diverse sexual identities, thus challenging the restricted dichotomy between masculinity and feminity. Such openness subverts the risk imaginary depicted by popular written media in both countries.

Key words: mass media, sociology of youth, sexual practices, social imaginaries.

\section{Sumario}

Palabras preliminares

1. El imaginario del riesgo

2. Socialización y subjetividad adolescente
3. Homosexualidad y respuesta adolescente a las ambigüedades identitarias

Palabras finales

Referencias bibliográficas

\section{Palabras preliminares}

El amplio consenso en las ciencias sociales en torno al rol protagónico que desempeñan los medios de comunicación en la socialización del sujeto permite comprender el interés que pueden suscitar sus representaciones de la sexualidad adolescente.

Teniendo presente que estos mismos medios promueven una economía cultural de mercado cuyos flujos de información nos vinculan a lo que García Canclini (2000) llama "la globalización imaginada», proponemos comparar las narrativas de las diversas expresiones de la sexualidad adolescente a partir de una muestra de diarios de amplia circulación en dos sociedades abiertas a procesos de cooperación e intercambio comercial e intercultural, pese a estructuras jurídico-políticas, tradiciones y niveles de desarrollo diferentes, como son Canadá y Chile. De acuerdo con Rebecca Sullivan y Kim Sawchuk (2006), partimos de la tesis que la construcción mediática de dicha sexualidad constituye un «actuar comunicacional», definido, a la luz de la teoría de Habermas (1987), como un proceso de negociación donde se afrontan y se reelaboran prácticas y discursos que, insertos en determinados contextos socioculturales, legitiman o, por el contrario, 
invisibilizan o desacreditan las vivencias cotidianas, tanto públicas como privadas, del deseo juvenil.

La revisión de dos periódicos canadienses (La Presse, publicación francófona de Montreal, y The Globe \& Mail, publicación anglófona de Toronto) y otros dos chilenos (El Mercurio y La Tercera) muestra la presencia de algunos ejes temáticos dominantes comunes y producciones de sentido subyacentes que instauran visiones particularizadas del tema e invisibilizan otras. Este efecto que orienta la mirada a ciertos aspectos de la sexualidad adolescente, contrasta con otro discurso de cierto reconocimiento social que anuncia una llamada "apertura» a la sexualidad estructurada en base a juicios como: "con la expansión de los procesos migratorios y la integración de los mercados e imaginarios de la comunicación, los ciudadanos miran hoy la sexualidad de una manera más abierta que ayer».

Se observa, sin embargo, un desfase entre esta percepción de «apertura» global y la casi inexistencia de un debate serio e informado en los mismos medios de comunicación respecto a temas como el aborto, el uso de anticonceptivos, las identidades sexuales, la relación entre amor y erotismo, así como diversos aspectos vinculados a la sexualidad en un sentido amplio, y no tan sólo a sus variantes reproductivas. Aunque en Canadá, en particular en las grandes urbes cosmopolitas como Montreal y Toronto, los medios parecen apropiarse de ciertas reivindicaciones de los movimientos queer y feministas, cabe interrogar el alcance y las ambigüedades de sus discursos supuestamente progresistas. Por su parte, tampoco en Chile resulta fácil encontrar opiniones suficientemente socializadas de instituciones relevantes como las iglesias, los partidos políticos o los grupos de intelectuales que hablen sobre el tema en cuestión.

La revisión a que hacemos referencia confirma que, más que una apertura real a una sexualidad considerada en sus manifestaciones psicosociales y culturales diversas, existe el predominio y la concentración temática en una visión catastrofista transnacional de la misma, donde el discurso mediático se ve monopolizado por la preocupación y el riesgo que implican las conductas sexuales diversas, más que en el goce y el placer que se puede encontrar en ellas mismas.

Surgen, así, ciertos ejes discursivos dominantes, como son: riesgos asociados a la práctica de la sexualidad, legitimación de la anticoncepción como reacción al abuso sexual o autoagresiones entre los adolescentes. Junto a ello, percibimos un vacío informativo en la medida que, a juicio de los diversos actores involucrados en esta temática (estado, profesores, padres, estudiantes), expresan poca claridad en torno a quién debe asumir un rol determinante en la producción y divulgación de estos contenidos. Frente al vacío, emerge una respuesta adolescente que, evidenciando las dudas propias de este estadio del desarrollo personal, al menos por el momento demuestra apertura a la existencia y manifestación de variadas identidades sexuales que sobrepasan la versión estrictamente dicotómica de lo masculino y lo femenino. Esto, por sí mismo, subvierte los criterios dominantes que recoge la prensa de mayor difusión en ambos países. 


\section{El imaginario del riesgo}

\subsection{Sexualidad «restringida»}

En el mes de abril del 2004, el diario El Mercurio hacía referencia a los diversos cambios y transformaciones que ciertos estudios demostraban que estaban ocurriendo en los últimos años en el comportamiento sexual y reproductivo de las adolescentes, destacando el aumento de la precocidad de las relaciones sexuales. Esta constatación supone un juicio que "subentiende» un tipo específico de relaciones sexuales, aquéllas de tipo genital y, la mayoría de las veces, con un miembro del sexo opuesto.

Esta concepción de la sexualidad soslaya una diversidad de sus manifestaciones de la cual dichos datos no dan cuenta, como son la relevancia de aquellos comportamientos autoeróticos en adolescentes de diverso sexo, por ejemplo, que también expresan formas de sexualidad en niveles de estructuración y desarrollo diferente o diversos tipos de caricias cuya meta no necesariamente son el coito heterosexual, el cual, una vez sublimado o simplemente reprimido, permite que estas caricias se puedan erigir en preámbulos de la formación sexual adolescente ${ }^{2}$. También deja de lado otras formas de expresión libidinal, como son las relaciones anales o el llamado sexo oral, conductas que parecen extenderse principalmente entre adolescentes, pues son percibidas por éstos como eficaces resguardos frente al contagio del virus del sida, las ETS o el embarazo no deseado.

En estrecha relación con lo anterior, se elaboran referencias a las posibles consecuencias de estas conductas, como lo es en particular la reproducción propiamente, más en concreto aún, los embarazos no deseados entre adolescentes, los cuales se mantienen en rangos bastante altos, pero, además, en los últimos decenios, acusan una disminución en la edad de ocurrencia. Según Rodríguez (2005), en 2002 se registraban 134,9 casos por cada 1.000 adolescentes. Una primera y lógica explicación, aunque de carácter general, de este hecho se encuentra justamente en que, al menos desde el plano de las probabilidades, si disminuye la edad de la primera relación heterosexual en los adolescentes, es esperable también una disminución en la edad de los embarazos en este rango etario.

Una situación epidemiológica semejante se observa en Canadá, donde los datos disponibles dan a entender que la edad media de la primera relación sexual disminuye constantemente. Se considera que el $18 \%$ de las adolescentes de 12 a 15 años han experimentado el sexo oral (Boekeloo y Howard, 2002) mientras que el $13 \%$ de las adolescentes ha tenido relaciones sexuales completas a la edad de 14 o 15 años. Al margen de estos nuevos patrones de comportamiento juvenil que llevaron al gobierno conservador federal a presentar, en junio de 2006, un proyecto de ley con miras a aumentar la edad legal del con-

2. «Originalmente, el organismo en su totalidad, y en todas sus actividades y relaciones, es un campo potencial para la sexualidad, dominado por el principio del placer» (H. MARCUSE, citado por EllIOT en Teoría social y psicoanálisis en transición. Buenos Aires: Amorrortu, 1995). 
sentimiento sexual de 14 a 16 años, Canadá dispone de tasas alarmantes de jóvenes con enfermedades de transmisión sexual. Por ejemplo, en el 2000, el $40 \%$ de los 32.869 casos de clamidia y el $41 \%$ de los 2.368 casos de gonorrea registrados a nivel nacional correspondían a chicas de 15 a 19 años (Agence de santé publique du Canadá, 2002). También se observa una feminización aumentada de casos seropositivos en la población adolescente. Y aunque los embarazos adolescentes han disminuido en los últimos años, el problema se ha agudizado en la población indígena de los territorios del norte.

Según datos de Statistique Canadá (2005), en estos territorios se da un 123,3 por mil de casos de embarazos de adolescentes (comparativamente, un 28,8 por mil en la provincia de Ontario), de los cuales un $45 \%$ terminan en aborto. En tal contexto, sorprende el poco interés de la prensa canadiense respecto a la SSR adolescente. Una excepción de ello la constituye una serie de tres artículos del Globe \& Mail de abril 2004 sobre la historia moderna de la planificación familiar y el rol crucial desempeñado por Simone de Beauvoir, Betty Friedan y otras pioneras de esta primera etapa de la «liberación sexual», incluyendo testimonios de mujeres «corrientes» decididas a ejercer el control sobre sus propios cuerpos.

Apelando a la multiplicación en el aumento de contraceptivos mejor adaptados a los diferentes ciclo de la fertilidad, el Globe \& Mail plantea abiertamente la cuestión de las desigualdades en salud sexual y reproductiva, en particular el hecho de que la contracepción aún se mantiene como una responsabilidad de las adolescentes y de las mujeres. Sus objetivos son alimentados por entrevistas emanadas de fuentes que ocupan posiciones de poder (cuerpo político, médicos y enfermeras), pero también de miembros de la sociedad civil, incluyendo ONG y ciudadanos sin pertenencia a ningún grupo determinado. Al contrario, del lado francófono se percibe un mutismo total en materia de SSR adolescente, incluidos los derechos a la contracepción. A menudo al límite de los problemas que nos ocupan, la ofensiva antifeminista recurrente en el periódico La Presse alimenta el cliché postmoderno del "desarraigo» de los hombres sin destino, supuestamente "domesticados», según la expresión de dicho diario, por la revolución feminista. También se desprende una denuncia de culpabilización injusta contra las madres de las prostitutas de 14 o 15 años y los grupos que asumen su defensa. Mientras que el argumento aquí consiste en relacionar la prostitución juvenil y la ausencia del padre (de hecho, las madres serían responsables de «la inmoralidad» de sus hijas), se invisibilizan los problemas de SSR, en particular la violencia sexual, el incremento de casos de sida, hepatitis y otras formas de ETS, a los cuales se enfrenta esta población adolescente (Durocher y otros, 2002).

\subsection{Legitimación contraceptiva frente al imaginario del riesgo: el caso de la pildora del día después ( $p d d$ )}

A diferencia del caso de La Presse, donde, como hemos comentado anteriormente, se elude el tema de la anticoncepción, la opinión que recoge el Globe \& 
Mail es claramente favorable al uso de la píldora del día después (pdd). Aunque en Canadá ésta se puede obtener sin prescripción médica, su acceso se ve parcialmente limitado por ciertas barreras, tales como la exigencia de consultar al farmacéutico de turno, pues éste puede o no distribuirla, sobre todo en aquellas comunidades rurales y remotas donde sólo existe una farmacia, así como las resistencias de grupos conservadores provida que asocian la pdd al aborto (Sibbald, 2005). Sin embargo, experiencias de numerosos países europeos, tales como Francia, Inglaterra, Dinamarca o Noruega, indican que la distribución de la pdd (sin prescripción médica ni intervención farmacéutica) no incide en el uso excesivo de la contracepción de emergencia (Sibbald, 2005).

En el caso chileno, el apoyo mediático a la pdd se limita a situaciones de abuso sexual. Mayoritariamente, el recurso a este método anticonceptivo es justificado en el caso de que la mujer haya sido víctima de violencia sexual. Esta opinión es compartida en diversos rangos etarios y su utilización tampoco constituye obstáculo para los propios padres de familia cuyas hijas puedan haber sido víctimas de algún tipo de abuso sexual. Cerca de un $80 \%$ de jóvenes chilenas considera útil y factible su utilización en el caso de violación, mientras que un $42 \%$, sólo si se rompe el condón, y un 33\%, si se olvida el anticonceptivo tradicional. Así lo consigna un estudio citado por uno de los medios analizados en este trabajo ${ }^{3}$.

Algunas autoridades edilicias opinan, de acuerdo con la Organización Mundial de la Salud, que la pdd puede ser distribuida para el uso de quien lo requiera cuando haya existido una relación sexual «no protegida y ante la falla de un método anticonceptivo, dejando abierta la posibilidad de legitimar la distribución y posterior utilización de la pdd de forma bastante generalizada». En este sentido, los medios analizados reflejan un cierto acuerdo entre los jóvenes, los padres de familia, las mujeres de distintas edades y entre diversas autoridades en torno al hecho de aceptar el uso de la pdd en estos casos. Esto último puede encubrir una posición latente, no manifiesta, relativa a la legitimación de su uso de forma más generalizada, dado que un abuso sexual no siempre puede ser comprobado efectivamente y, además, las personas víctimas de abuso no recurren a su demostración y constatación por personal especializado. Podemos, así, estar en presencia del recurso a una justificación "socialmente aceptada» para prevenir de manera artificial un posible embarazo no deseado.

En la actualidad, el uso de la pdd no ha incidido en el uso normal de otros métodos anticonceptivos. Es más significativa, sin embargo, a través de su uso, la poca participación del hombre en la anticoncepción, pues si bien el condón requiere de su participación activa en este proceso, el uso de la pdd reinstala de forma más específica esta acción en un ámbito decisional de la mujer, con lo que se contribuye a reforzar un modelo de feminización de la anticoncepción.

3. Estudio del Centro de Medicina Reproductiva y Desarrollo Integral del Adolescente (Ceinera). Facultad de Medicina, Universidad de Chile (citado por La Tercera, abril de 2004). 
Incorporar de forma activa al hombre en el cuidado y la precaución del embarazo especialmente adolescente y no deseado puede constituir un refuerzo significativo de aquellas conductas precautorias en este plano, dado que se produce así una decisión de pareja y no unilateral, como ocurre mayormente en la actualidad, puesto que se otorga la decisión, la culpa y la responsabilidad en el ámbito primordialmente de la mujer.

\subsection{Abuso sexual y autoagresión adolescente}

A diferencia de los piercings (perforaciones) y de los tatuajes, que pueden representar una suerte de rito de iniciación o de paso a la edad adulta, la automutilación se manifiesta en actos deliberados de violencia autoinflingida que comportan heridas corporales, entre otros comportamientos (físicos y emocionales) autodestructores, como el abuso de substancias y conductas de riesgo sexual. En el plano físico, la automutilación puede adoptar varias formas: cortarse la piel con una hoja de afeitar o con un trozo de vidrio, quemarse, darse golpes, rasguñarse, sacarse pedazos de piel para impedir que sanen las heridas, tirarse el cabello e insertarse objetos en el cuerpo. En Canadá, los cortes representan la forma más común de automutilación entre los jóvenes. Varios de ellos discuten de sus cortes por Internet, algunos llegan incluso a formar clubes de heridos voluntarios en el colegio. Si bien en las sociedades occidentales estas prácticas no son un fenómeno nuevo, éstas se han venido haciendo de forma cada vez más frecuente. Una encuesta canadiense revelaba que alrededor del 13\% de adolescentes manifestaban comportamientos de automutilación (Association canadienne pour la santé mentale, 2007).

En Estados Unidos, se estima que la incidencia de automutilaciones casi se ha doblado en veinte años, mientras que, en Francia, este fenómeno parece afectar a algunas decenas de miles de jóvenes por año, principalmente niñas, y de manera más precoz (Lasterade, 2006). Como se trata de una actividad secreta, resulta muy difícil determinar con exactitud la cantidad de jóvenes que se automutilan.

Un estudio de la Association canadienne pour la santé mentale (2007) deja entender que la mayor parte de las mujeres jóvenes que se automutilan surgen de la clase media o superior, tienen inteligencia media o superior, pero demuestran una débil autoestima. Mientras un $40 \%$ de ellas ha conocido problemas alimenticios (anorexia o bulimia), cerca de la mitad afirma haber sido víctima de agresión física o sexual durante su infancia. La mayor parte reconoce tener dificultades para expresar sus emociones, particularmente la cólera y la tristeza. Otras investigaciones (Adams, Rodham y Gavin, 2005; Fillmore y Dell, 2001; Huntington, 2001) confirman que los primeros episodios de automutilación están ligados a agresiones y a violencia sufrida durante la infancia.

No resulta fácil encontrar una explicación racional para el abuso sexual y las autoagresiones en que incurren las adolescentes, como lo señalan los medios. Podemos reconocer la existencia de ciertos condicionantes sociales que actúan 
en forma contradictoria, al establecer, por una parte, el predominio de una cultura hedonista erotizada a través del cine, la televisión, las revistas o las canciones, por mencionar algunas manifestaciones de la cultura y, por otro lado, una información mediática sobre sexualidad que apunta en forma prioritaria a presentarla bajo el manto de la amenaza del contagio, la infección o el embarazo no deseado, soslayando la perspectiva centrada en el goce y el placer, que, por cierto, también conforman la sexualidad humana.

De esta forma, las adolescentes reciben múltiples mensajes que les estimulan a lograr la meta sexual, el coito, pero simultáneamente se les reprime dicho estímulo. Así, el deseo estimulado culturalmente, por una parte, y la inhibición simultánea del mismo por la vía del temor a su concreción, son dos caras de una misma moneda en el contenido mediático en la actualidad.

La desinformación respecto a las relaciones de poder y control que inciden en las agresiones sexuales conduce a una narrativa del temor, en línea con el imaginario del riesgo, donde irrumpen metáforas del crimen y estereotipos del "sexo débil». Se puede suponer también que, al invocar el clima de inseguridad social provocado por el supuesto aumento de la delincuencia y la «decadencia» de los comportamientos sexuales, los medios alimentan el miedo al otro. Es así como discriminaciones de género, de clase, de etnia o de raza encuentran un terreno fértil.

Otro factor explicativo de estos comportamientos dentro de una perspectiva sociológica es la creciente validación social de los mismos. A ello contribuye una moda promovida por los medios de comunicación, como son los relatos de artistas famosos (Marilyn Manson, por ejemplo), que reconocen y publicitan sus comportamientos autoflagelantes y la reafirmación de lo masculino, en el contexto latinoamericano, expresado en la tolerancia máxima al dolor como expresión de un machismo exacerbado, aunque esta conducta también se reconoce en las mujeres, como mencionamos anteriormente. Si bien estos testimonios mediatizados se benefician de un aura particular, ellos estimulan a ciertas adolescentes vulnerables a querer copiar los comportamientos autoagresivos.

En el decir de Bourdieu, se subjetiviza la frustración de una sexualidad autorreprimida y violentada en relación con los sistemas y las estructuras sociales en general ${ }^{4}$, sobre todo cuando estas autoagresiones (o automutilaciones) ocurren en jóvenes que, por lo general, no padecen de trastornos psíquicos severos.

Las investigaciones feministas, por su parte, proponen una explicación alternativa. Sus estudios retoman la tesis de M. Foucault, según la cual el cuerpo es, a la vez, un sitio de control y de resistencia donde se cristalizan las relaciones de poder (biológicas, psicológicas y sociales) que intervienen en diversos niveles. Como señala Sylvie Frigon (2000), el primer nivel es el de la ritualización, definido como el conjunto de prácticas que producen la apa-

4. Para una relación entre sistema psíquico y sistema social, se puede consultar DE GAULEJAC, V. Sociologie Cliniques. También se puede revisar el concepto de «habitus» en Bourdieu. 
riencia corporal como signo: adornos del cuerpo, máscaras, hábitos, posturas, gestos. Luego, se superpone un segundo nivel que comprende las prácticas de reproducción del cuerpo, su manutención cotidiana en sus dimensiones biológicas y sociales (por ejemplo, la salud o el adelgazar). En un tercer nivel, estructural o simbólico, se elaboran las estrategias de reapropiación de la identidad.

Como expresión de estas estrategias, la automutilación aparece como un medio privilegiado de resistencia en la medida que ella permite reapropiarse del propio cuerpo. Para algunas adolescentes, cortarse o hacerse heridas se transforma en una manera de ejercer un cierto control sobre sí mismas.

Siguiendo a Frigon (2000: 146-147), gracias a los procesos de automutilación, "las mujeres pueden contar sus vidas a través de las marcas de laceración». En el interior del cuerpo marcado habrá una «doble construcción del cuerpo de las mujeres, el cual es considerado en peligro y peligroso, como un cuerpo víctima (objeto de dominación) y un cuerpo agresor (sujeto de resistencia)».

En resumen, la automutilación constituiría un mecanismo de adaptación y de sobrevivencia permanente, una manera de hacer frente al dolor emocional, a la angustia, al aislamiento y a las situaciones opresivas, por ejemplo: a la violencia. Ella aportaría a los adolescentes un «sentimiento de liberación, purificación y control» (Centre canadien de lutte contre l'alcoholisme et les toxicomanies, 2006, p. 2). A pesar de su carácter insano, permitiría, entonces, la afirmación de la experiencia vivida de la subjetividad, es decir, la capacidad de actuar y de sufrir por sí mismo.

Las autolesiones como conducta emergente aunque transversal en nuestra sociedad responde, así, a una tríada causal de carácter biopsicosocial. De allí la pertinencia que, en su control y tratamiento, intervengan equipos interdisciplinarios, con el fin de encontrar una salida integral a este nuevo desafío que nos impone la sociedad actual.

\section{Socialización y subjetividad adolescente}

\subsection{La "desinformación» juvenil sobre sexualidad}

La revisión de los cuatro medios que conforman este análisis pusieron en evidencia justamente que, en relación con la salud sexual y reproductiva de los adolescentes, los aspectos que parecen monopolizar un escaso diálogo al respecto son restrictivamente aquéllos vinculados a la reproducción, olvidando casi por completo cualquier otra dimensión de la sexualidad humana que no tenga relación directa con la reproducción y las ETS, lo que desmiente la supuesta "apertura» de la opinión pública para mirar su sexualidad. El propio análisis de los medios demuestra esto último.

Un aspecto importante que destacan los medios canadienses y chilenos al tratar este tema es que, si bien reconocen, según la opinión de especialistas, que las jóvenes reciben una buena cantidad de información sobre su sexualidad, 
ésta no es aplicada en la práctica ${ }^{5}$. En la información, predomina el interés por estimular una conciencia más bien autorrepresiva y precautoria ante el deseo sexual adolescente, que una visión informada sobre los modos de realizar este deseo de manera responsable y con respeto mutuo en la pareja.

Otra explicación de la indiferencia que manifiestan los adolescentes frente a este tipo de información, como lo muestran estudios realizados a nivel latinoamericano, son componentes de carácter sociocultural, como los criterios que evidencian la masculinidad en el hombre, los cuales adquieren mayor influencia entre los jóvenes que los indicadores de riesgo para su salud, al adoptar conductas desprotegidas en el plano sexual. La información sobre sexualidad se enfrenta, así, a la construcción efectiva de criterios que definen la identidad de género presente en las cosmovisiones socioculturales de los adolescentes.

Por su parte, las propias autoridades de la salud reconocen que no existen espacios adecuados donde se escuche abiertamente a las jóvenes. Aunque, en Canadá, el Gobierno federal ha establecido una serie de iniciativas proactivas (incluyendo recursos adicionales para las clínicas de atención a adolescentes "en riesgo", la creación de sitios web dirigidos a las adolescentes y a sus padres, así como el respaldo de los esfuerzos provinciales en materia de educación sobre SSR), un estudio realizado por el Ministerio de Salud confirma que la diversidad de expresiones de la sexualidad juvenil aún carece de la atención pública requerida (Santé Canadá, 2003a: 50).

El problema se complejiza en Chile, dado que no existe una predisposición, al menos de parte de los servicios públicos de salud, para atender las profundas y reales preocupaciones de la población adolescente en estas materias. Su gran falencia es el hecho de no disponer justamente de una información que otorgue respuestas integrales a las inquietudes juveniles. Esto último da cuenta, así, de una información que el joven percibe sesgada o al menos incompleta, incapaz de satisfacer sus requerimientos acerca de un conocimiento más acabado de la materia en cuestión.

En el caso chileno, la respuesta de las autoridades ha consistido en parte en elaborar manuales de información, a partir de las inquietudes manifestadas por los adolescentes en actividades que forman parte de un programa implementado en diversas comunas por el Ministerio de Educación. Estos manuales intentan recoger las demandas expresadas por los estudiantes en cuanto a su necesidad de poder hablar sobre sexualidad libremente y sin recriminaciones de los adultos.

A lo anterior, podemos agregar las carencias del diálogo y de una efectiva comunicación entre padres e hijos al respecto, donde, de existir, será bajo los parámetros predeterminados por un paradigma restrictivo de la sexualidad adolescente, en el cual, como señalamos, ésta aparece vinculada esencialmente a las conductas de riesgo para la salud. En otras palabras, y como parecen demostrar los medios, la mayor apertura a la sexualidad es deudora de una perspectiva catas-

5. Una expresión de ello es que, como se destaca en un reportaje, a pesar de la información disponible, sólo el 24,8 \% de los jóvenes entre 18 y 24 años usó un método anticonceptivo cuando se inició sexualmente. 
trofista de la misma ${ }^{6}$, con lo cual se conforma un vacío en la formación integral del adolescente sobre su propia sexualidad y el conocimiento de sí mismo.

\subsection{El vacio institucional como agente de socialización}

Para los medios analizados, una consecuencia de lo anterior se puede encontrar en el hecho que entre aquellas adolescentes con menor educación ocurre con mayor frecuencia el inicio precoz de las relaciones sexuales, lo que provoca un mayor número de embarazos no deseados y el recurso al aborto como método para enfrentarse a esta situación. No se trataría tanto de una disponibilidad de recursos económicos lo que provocaría estos efectos, sino la carencia de una información adecuada.

Este planteamiento induce a pensar que la forma de enfrentarse a esta problemática debería ser multifacética, dado que el éxito de una estrategia de parte de las autoridades comprometidas en SSR no dependería únicamente del énfasis puesto, por ejemplo, en la divulgación o la distribución masiva de métodos anticonceptivos, cuyo acceso introduce la variable económica de por medio (disponibilidad de recursos para su adquisición), sino que en este aspecto también serían importantes el ámbito educativo y el de la información.

Por tanto, parte de la responsabilidad para afrontar lo anterior recae principalmente en los agentes encargados de la educación de las adolescentes: los padres y la escuela en forma preferente. Estudios del Mineduc demuestran que existe efectivamente una suerte de acuerdo colectivo mayoritario entre los diversos actores del sistema educativo (autoridades ministeriales, padres y apoderados, profesores y alumnos, éstos últimos en menor medida) en torno a que son los padres los primeros responsables de formar a sus hijos en este ámbito, lo cual contradice una realidad familiar que, en la actualidad, evidencia la ausencia del padre y de la madre del hogar, principalmente por razones laborales ${ }^{7}$.

Por otra parte, una participación definitoria de contenidos y sentidos dominantes en este plano de parte de la autoridad pública resulta impensable, pues, en el decir de J. Habermas «esto pondría en aprietos a un Estado que está obligado a mantener la neutralidad en lo que se refiere a cosmovisiones, precisamente en atención al hecho del pluralismo» ${ }^{8}$. Lo anterior,

6. Mientras que, en Canadá, las manifestaciones homofóbicas (insultos o agresiones verbales, violencia física) y el aumento de solicitudes de asilo político debido a violaciones de derechos de las personas en razón de su orientación sexual retiene la atención de los medios, sus análisis sobre la discriminación, la victimización de gays y lesbianas se mantienen muy superficiales. Por otra parte, las necesidades de salud sexual de esta población se presentan exclusivamente en un contexto epidemiológico de riesgo, donde la pandemia del sida adquiere un lugar cada vez más importante en el imaginario colectivo.

7. Esta realidad se agudiza en el caso de los numerosos hogares donde la mujer ha asumido el rol de jefa de su casa y donde los hijos, en la mayoría de los casos, quedan bajo el cuidado de una hermana mayor, una vecina o, simplemente, una asesora del hogar.

8. HABERMAS, J. «Razón secular y religión en el Estado moderno». Ponencia leída en el encuentro Sobre las bases morales prepolíticas del Estado liberal, enero de 2004. 
entonces, deja "en tierra de nadie» la responsabilidad, tanto por la definición de contenidos y sentidos orientadores de la educación sexual adolescente, como la identificación de los agentes encargados de su implementación efectiva. En realidad, sabemos también por diversos estudios (Mineduc, 2004) que dicha educación no queda en el vacío, sino que éste es llenado en gran medida por el "grupo de pares»: son las amistades de edades próximas las que están socializando principalmente a los adolescentes en estas materias. Esto último supone un orden social y una forma de relaciones entre los padres y el sistema educativo de carácter ilusorio que reproduce una indefinición de roles cuyo efecto se traduce en la liberación de responsabilidades a ambos actores.

\section{Homosexualidad y respuesta adolescente a las ambigüedades identitarias}

\subsection{Identidades sexuales y hegemonías mediáticas}

En los dos contextos analizados, la prensa vehicula la idea de una cierta apertura de parte de los jóvenes respecto de la homosexualidad. Sin embargo, en ningún caso, ésta reconoce la opinión de gays y lesbianas, padres o niños, en tanto que interlocutores. Lejos de desmantelar los prejuicios homofóbicos, más bien parece que los periódicos refuerzan las identidades sexuales hegemónicas en detrimento de aquéllas que cuestionan o desorganizan las oposiciones binarias de lo considerado socialmente «normal o anormal». En la prensa canadiense, el debate sobre la libertad de orientación sexual se articula principalmente en torno a proyectos legislativos guiados por las cartas quebequense y canadiense de derechos y libertades. Los principales temas abordados son los nuevos lazos de filiación autorizados en virtud de la ley sobre la unión civil adoptada en Québec en 2002 y el proyecto de ley del gobierno federal (adoptada en 2005) sobre el reconocimiento jurídico de las uniones de cónyuges del mismo sexo. Así, se enfrentan posiciones progresistas, representadas por el Gobierno federal y las ONG (Gai Écoute, Égale Canada, Coalition des Canadiens pour un mariage égal), y aquéllas de grupos conservadores católicos opuestos a los cambios legislativos en la materia.

$\mathrm{Si}$, por otra parte, el interés por la procreación asistida conduce a análisis sobre las consecuencias, es decir, los «riesgos» de la maternidad lesbiana, su procedimiento se asemeja a los de los medios chilenos. Para brindar consistencia al tema, los diarios dan cuenta de investigaciones basadas en entrevistas a niños de padres gays o de madres lesbianas que dan testimonio de una denominada "tolerancia» de los jóvenes frente a la identidad sexual de sus padres, subrayando sus "temores» concernientes a la eventual estigmatización que ellos podrían sufrir de parte de sus pares.

Más allá de la carga propiamente afectiva de estos procedimientos periodísticos, es la lógica de la "domesticación de la diferencia», según la expresión de Juan Pablo Sutherland (2004, p. 123), lo que permite a los medios una rea- 
propiación doctrinal de las singularidades «subalternas», en este caso homosexuales, destinadas a validar las orientaciones y los comportamientos sexuales acordados en el plano moral.

Quizás si la mayor apertura de las adolescentes al tratamiento o consideración no excluyente de las relaciones homosexuales entre personas genitalmente femeninas o masculinas encuentre su explicación en una postura de atención a la ambigüedad o indefinición que suponen esos caracteres y a la aceptación que la realidad en diversas manifestaciones se resiste a verse encasillada en perspectivas dicotómicas del todo o nada. Está claro que, al menos desde una aproximación cultural postmodernista, estas «identidades sexuales fronterizas» (Martínez, Meneses y Sarabia, 2005) representan un cuestionamiento o una afrenta a cierta mirada que se vierte sobre la sexualidad humana enmarcada en el estereotipo de los polos opuestos.

\subsection{Subversión imaginaria de los paradigmas identitarios dominantes}

La aceptación, entonces, de la homosexualidad por parte de los adolescentes puede ser considerada como un ejercicio de subversión imaginaria de los estereotipos de una cultura eminentemente racionalista y obsesiva que, frente a la ambigüedad o a la falta de imaginación, recurre a definiciones restrictivas de la realidad.

Desde el reduccionismo singular de la perspectiva de género que encasilla las identidades en dos términos, como si la edad, la clase o la raza no tuviesen nada que decir en esta definición o si la historicidad de este constructo cultural tampoco tuviese valor explicativo, la construcción dicotómica de los sexos resulta más comprensible como mecanismo de autodefensa que como epistemología de la sexualidad.

Si la postmodernidad se ha identificado como la era del relativismo no exclusivamente moral, como lo han querido catalogar los espíritus más conservadores, sino, desde una perspectiva positiva como expresión de la duda permanente y el estímulo al autocuestionamiento del orden de las significaciones adheridas a los significantes dominantes, es posible considerar que estemos frente a un adolescente que mira, aún con dudas y temores por cierto, pero que se abre a la consideración de una naturaleza de la sexualidad distinta a la predominante hasta ahora.

Esta mirada abierta a una mayor diversidad en cuanto a identidades sexuales conlleva, a la vez, el reconocimiento de los diversos caminos que puede seguir la afectividad entre las personas. Y es que el indicador genital al que recurre la cultura para distinguir al hombre de la mujer, no siempre ni necesariamente equivale o se corresponde con una postura psíquica que defina los afectos en el mismo sentido unidireccional de la intersubjetividad, como lo hace el paradigma de la genitalidad o del origen biológico de los sexos.

Desde esta perspectiva, el discurso de los poderes públicos que insiste en una definición dicotómica de la sexualidad, da muestras de mantenerse anclado en visiones desapegadas del mundo adolescente con las cuales, ciertamen- 
te, este rango etario no se identifica. Es probable, así, que el discurso educativo en estas materias debiera más bien ofrecer oportunidades para tratar los temas relativos a las definiciones de identidades sexuales, por ejemplo, en lugar de enfatizar casi de forma exclusiva aquellos aspectos de la sexualidad adolescente relacionados con los resguardos necesarios para evitar una sexualidad «riesgosa».

\section{Palabras finales}

En resumen, a pesar de que los medios dan testimonio de las tensiones sociales específicas de sus propias sociedades (lucha por la distribución gratuita de la píldora del día después en Chile, el matrimonio de personas del mismo sexo en Canadá y en Québec), las normas dominantes de lo «aceptable» obedecen a una lógica homogeneizante del miedo y el riesgo que limita considerablemente el debate en materia de sexualidad adolescente. Hemos visto que, independientemente de su inserción geopolítica, social o cultural, los diarios estudiados se muestran incapaces de pensar en cuidar el cuerpo sin ahogar la afirmación identitaria de las «subjetividades disidentes» emergentes.

Como señala Chantal Nadeau (2006), a pesar de las tentativas repetidas de la teoría queer de imaginar y de articular un discurso con un sujeto por debajo de la ley, aparece siempre la tiranía de un cuerpo que, incluso sometido a innumerables tensiones, se enfrenta en un espacio público a no ser de hecho más que un cuerpo parcelado donde cada parte es fácilmente identificable a través de los parámetros dominantes: aquél de categorías identitarias comprobadas (p. 922).

Este parcelamiento del cuerpo es tan manifiesto que, sistemáticamente, hace abstracción de la intersección de sistemas de poder — de género, de orientación sexual, de clase, de "raza» y/o de etnia- que moldean la pluralidad de sexualidades. Sabiendo que los pueblos indígenas representan alrededor del $3 \%$ y del $4,6 \%$ de la población total de Canadá y de Chile, respectivamente, nos podemos asombrar como pueden ser ocultadas las necesidades y las experiencias de jóvenes autóctonos. De hecho, la alteridad sexual, ya sea autóctona, mestiza o de color, se enfrenta al rígido cuadro de tabús y otras prohibiciones del amplio espacio público mediático. Incluso el deseo se encuentra confinado en un silencio que obstaculiza todo aquello que recuerda, de cerca o de lejos, el placer erótico y su torrente pulsional: heterosexualidad, homosexualidad, bisexualidad o transgénero. Se puede esperar que la mayor apertura de los jóvenes a la diversidad de identidades sexuales ponga en jaque a las restrictivas explicaciones "oficiales» de una sexualidad que se muestra aún inexplorada en múltiples facetas y en la cual nos obligan a seguir reconociendo su profunda y enigmática naturaleza. 


\section{Referencias bibliográficas}

Adams, J.; Rodham, K. y Gavin, J. (2005). "Investigating the "self» in Deliberate self-harm». Qualitative Health Research, vol. 15 (10), p. 1293-1309.

AGENCE DE SANTÉ PUBLIQUE DU CANADA (2002). "Rapport de surveillance canadien 2002 sur les infections transmises sexuellement» (suplemento). Relevé des maladies transmissibles au Canada, vol. 31S2.

Aguirre, R. y GÜELL, P. (2002). Hacerse hombres: La construcción de la masculinidad en los adolescentes y sus riesgos. OPS-OMS.

ASSOCIATION CANADIENNE POUR LA SANTÉ MENTALE (2007). Les jeunes et l'automutilation [en línea]. <http://www.cmha.ca/bins/content_page.asp?cid=3-1036\& lang $=2>$

BoEKelOO, Bradley O. y Howard, Donna E. (2002). «Oral sexual experience among young adolescents receiving general health examinations». American Journal of Health Behavior, 26(4), 306-314.

Bourdieu, P. (2000). La dominación masculina. Barcelona: Anagrama.

CENTRE CANADIEN DE LUTTE CONTRE L'AlCOOLISME ET LES TOXICOMANIES (2006). L'automutilation chez les femmes criminalisées [en línea]. <http://www.ccsa.ca/NR/ rdonlyres/7DB504EE-6387-4EAE-80F8-7E368BB6014E/0/ccsa0113392006f.pdf>

Delval, J. (1999). Desarrollo humano. Madrid: Siglo XXI.

Documento de trabajo de la Comisión Consultiva para la Revisión y Actualización de la Politica de Educación en Sexualidad del Ministerio de Educación. Santiago de Chile, 2004.

Durocher, L.; Fleury, É.; BerThiaume, P. y MoÏSE, J. (2002). «La prostitution juvénile, quoi de neuf?». Revue Défi Jeunesse, núm. 9 (1): 23-30.

Elliot, A. (1995). Teoría social y psiconálisis en transición. Buenos Aires: Amorrortu. Fillmore, C. y Dell, C. (2001). Prairie women: Violence and self-harm. Winnipeg: Société Elizabeth Fry du Manitoba.

Foucault, M. (2002). Historia de la sexualidad. Buenos Aires: Siglo XXI.

FrigOn, S. (2000). "Corps, féminité et dangerosité: de la production de «corps dociles» en criminologie», capítulo 4. Du corps, des femmes. Contrôles, surveillances et résistances (dirección de Sylvie Frigon y Michèle Kérisit). Ottawa: Les Presses de l'Université d'Ottawa. Études des femmes.

García CanClini, N. (2000). La globalización imaginada. Buenos Aires, Barcelona, México: Paidós.

GIDDENS, A.(1992). La transformación de la intimidad. Madrid: Cátedra.

Habermas, J. (1987). Théorie de l'agir communicationnel. Primer tomo: Rationalité de l'agir et rationalisation de la société. París: Fayard.

HunTington, A. (2001). "Childhood maltreatment and adult self-injury: A woman's account». Practice (R.-U.), vol. 13(2), p. 31-42.

LASTERADE, J. (2006). "Anorexie, boulimie, automutilation et adolescence». Journal Libération, 24 de noviembre [en línea]. <http: //www.liberation.fr/actualite/ societe/219054.FR.php>

Le Breton, D. (2002). La sociología del cuerpo. Buenos Aires: Nueva Visión.

Mineduc. «Documento de Trabajo de la Comisión Consultiva para la revisión y Actualización de la Política de Educación en Sexualidad del Ministerio de Educación», junio 2004.

Martínez, A.; Meneses, A. y Sarabia, D. (2006). «La représentation de la sexualité adolescente dans les quotidiens du Canada central et du Chili: quelles leçons tirer des Conférences du Caire et de Beijing?». Canadian Journal of Communication. 
Edición especial Sexe/Sexualités, bajo la dirección de Kim Sawchuk y Rebecca Sullivan, vol. 31, p. 881-896.

MarTíneZ, A.; MENESES, A. y SARABIA, D. (2005). «La educación de la salud sexual a $1 @ s$ adolescentes en Canadá y Chile: una mirada desde las políticas públicas». Revista Chilena de Temas Sociológicos, núm. 10. Ediciones UCSH: Justino Gómez de Benito y Mario Sandoval Manríquez, p. 49-99.

Minsky, R. (1998). Psicoanálisis y cultura. Valencia: Frónesis.

Morel, G. (2002). Ambigüedades sexuales. Buenos Aires: Manantial.

NADEAU, C. (2006), «L'urgence-désir comme engagement». Canadian Journal of Communication, Special Issue: Sexualities, vol. 31, núm. 4, p. 919-928.

Rodríguez, J. (2005). "Adolescent reproduction: the case of Chile and its policy implications». Cepal Review, núm. 86: 117-140.

SANTÉ CANADA. (2003). Lignes directrices nationales pour l'éducation en matière de santé sexuelle. Ottawa, Santé Canada, 2003. Publié à http://www.hc-sc.gc.ca/pphbdgspsp/publicat/cgshe-ldnemss/emss_index.html <http://www.hc-sc.gc.ca/pphbdgspsp/publicat/cgshe-ldnemss/emss_index.html>.

SiBBALD, B. (2005). «Nonprescription status for emergency contraception». Canadian Medical Association Journal, núm. 172(7): 861-862.

STATISTIQUe CANADA (2005). Indicateurs de la santé, núm. 2.

Sullivan, R. y SAWCHUK, K. (2006). «Editorial». Canadian Journal of Communication, Special Issue: Sexualities, vol. 31, núm. 4, p. 781-789.

SUTHERLAND, J. P. (2004). «Traductibilidad y proyección política: la sistematización y politización de los saberes y/o su des-politización». Hacia una agenda sobre sexualidad y derechos humanos en Chile. Editores: Teresa Valdés y Gabriel Guajardo. Chile: FLACSO, p. 123-130. 